\title{
DESPENALIZACIÓN DEL ABORTO EN CHILE. UNA CUESTIÓN DE JUSTICIA SOCIAL
}

\author{
Claudia Donoso Sabando ${ }^{1}$
}

Resumen: Las posturas respecto de este tema se mueven en un continuo que va desde los movimientos ultraconservadores hasta los más liberales. En este contexto, establecer políticas sanitarias sobre la base de sistemas morales absolutos no resulta política ni éticamente correcto en sociedades pluralistas como la nuestra. Por lo mismo, urge establecer unos acuerdos mínimos que permitan el desarrollo de los proyectos de vida feliz de cada cual. Chile, con una política fuertemente antiabortista, necesita revisar sus legislaciones con el fin de asegurar una atención justa a todos sus ciudadanos. Los datos estadísticos revelan que la prohibición no elimina la práctica, más aún, coloca en una condición francamente disminuida a los que ya presentan una situación de desventaja producto de la "lotería social de la vida". Poner atención a este tema y actuar de una vez manifestando de manera tangible lo que tantas veces se ha conversado en nuestro país, es una deuda de justicia que el estado chileno tiene con todas las mujeres.

Palabras clave: aborto inducido, justicia social, derechos de la mujer

\section{Decriminalization of abortion in Chile. A question of social justice}

Abstract: The positions on this issue move on a continuum ranging from ultra-conservative to the most liberal movements. In this context establishing health policies based on absolute moral systems doesn't result in political or ethical correctness in pluralistic societies like ours. For this reason, it urges the establishment of minimum agreements that allow the development of happy life projects for every person. Chile, which has a strong anti-abortion policy, needs to review their laws to ensure fair attention to all citizens. The statistics reveal that prohibition does not eliminate the practice of abortions; furthermore, it puts people who are already disadvantaged due to "the social lottery of life" in a worse situation. Paying attention to this issue and acting immediately showing in a tangible way that which has been discussed many times in our country, is a debt of justice that the Chilean state has to all women.

Key words: abortion induced, social justice, women's rights

\section{Despenalização do aborto no Chile. Uma questáo de justiça social}

Resumo: As posturas a respeito deste tema se movem num contínuo que vai desde os movimentos ultraconservadores até os mais liberais. Neste contexto, estabelecer políticas sanitárias sobre a base de sistemas morais absolutos não resulta política nem eticamente correto em sociedades pluralistas como a nossa. Por isso mesmo, urge estabelecer alguns acordos mínimos que permitam o desenvolvimento dos projetos de vida feliz de cada qual. O Chile, com uma política fortemente anti-abortista, necessita revisar suas legislaçóes com a finalidade de assegurar uma atenção justa a todos seus cidadãos. Os dados estatísticos revelam que a proibição não elimina a prática, mais ainda, coloca numa condição francamente diminuida aos que já apresentam uma situação de desvantagem produto da "loteria social da vida". Colocar atenção neste tema e atuar de uma vez manifestando de maneira tangível o que tantas vezes se tem conversado em nosso país, é uma dívida de justiça que o estado chileno tem com todas as mulheres.

Palavras-chave: aborto induzido, justiça social, direitos da mulher

\footnotetext{
${ }^{1}$ Departamento de Estomatología, Facultad de Ciencias de la Salud, Universidad de Talca, Chile. Correspondencia: cldonoso@utalca.cl
} 


\section{Introducción}

La cuestión del aborto constituye una de las problemáticas clásicas en del campo de la bioética. Los valores en conflicto se sustentan principalmente en torno a dos hechos; por un lado, la protección contra el daño de la vida del que está por nacer, sustentado por el principio de no maleficencia, y, por otro, el respeto al ejercicio de la autonomía de la madre sobre el control de su salud sexual y reproductiva. Los movimientos radicales a favor o en contra de esta práctica ven dicha conflictividad de valores dilemáticamente, entregando respuestas disyuntivas. Lógicamente, una adhesión absoluta a cualquiera de estas dos visiones no solucionará la controversia; resulta imperioso encontrar un punto intermedio para transformar este dilema en problema y, con base en el diálogo de todos los involucrados, hallar un acuerdo mínimo que permita a las personas desarrollar sus proyectos de vida buena.

Actualmente, Chile es uno de los cinco países en el mundo que reclama una prohibición absoluta frente a la práctica del aborto. Esta situación ha ido creando a través de los años una serie de problemas sociales y sanitarios que es necesario enfrentar prontamente. En los siguientes párrafos se intentará explicar por qué la figura de la despenalización del aborto en nuestro país constituye un asunto de justicia social. Primeramente se expondrán los hechos en cuanto a términos, cifras y opinión pública, para justificar con datos estadísticos los problemas sociales y sanitarios que involucra la penalización absoluta del aborto. Luego, las implicancias de género en el tema, ya que si el embarazo se desarrolla en el cuerpo de la mujer, es interesante comprender el contexto social en el cual ella está inmersa y así vislumbrar, aunque sea brevemente, las razones y consecuencias de un embarazo "a la fuerza". A continuación, una perspectiva acerca de la condición ontológica del embrión, debate clásico e interminable, pero muy importante para puntualizar la postura y los motivos que podrían justificar la realización de un aborto cuando las condiciones lo ameriten, y finalmente la exposición de las razones de por qué la despenalización del aborto constituiría una figura desarrollada dentro al área de los mínimos morales, particularmente en torno al principio de justicia.

\section{Lo primero, los hechos}

Para contextualizar el tema tanto desde el punto de vista de las definiciones como de los datos estadísticos en referencia al aborto, se optó por tratar el asunto desde los antecedentes obtenidos a través de la Organización Mundial de la Salud (OMS), pues esta constituye un referente transversal para los distintos países y presente en la diversas publicaciones aplicadas a este tema. Los datos de Chile se obtuvieron de acuerdo a lo dispuesto por el Ministerio de Salud.

La Organización Mundial de la Salud define "aborto" como "la interrupción de un embarazo tras la implantación del huevo fecundado en el endometrio antes de que el feto haya alcanzado viabilidad, es decir, antes de que sea capaz de sobrevivir y mantener una vida extrautaerina independiente. Esta interrupción puede ser espontánea o provocada”(1).

Las condiciones por las cuales el aborto es permitido varían de un país a otro. Las últimas cifras oficiales de la OMS indican que, en el mundo, de un total de 193 países, el 98\% de los países permite el aborto para salvar la vida de la mujer, $67 \%$ para preservar la salud física, 65\% para preservar la salud mental, $49 \%$ por violación o incesto, $46 \%$ por malformaciones fetales, $34 \%$ por razones económicas o sociales, y un $28 \%$ por requerimiento de la madre(2). En aquellos países donde el aborto se encuentra penalizado - como ocurre en Chile- o fuertemente restringido se presenta la figura del aborto inseguro o también llamado "aborto provocado en condiciones de riesgo", definido por la OMS como "aquel procedimiento para terminar un embarazo no deseado practicado por personas que carecen de las calificaciones necesarias o que es practicado en un ambiente sin las mínimas condiciones de seguridad médicas, o ambos" (2).

El último informe respecto de este tema entregado por la OMS afirma que en 2008 se realizaron en el mundo un promedio de 21,6 millones de abortos no seguros, la mayoría en países en vías de desarrollo. Asimismo, la tasa promedio mundial de aborto inseguro por cada 1.000 mujeres entre 15 y 44 ańos fue de 14(2). Además, del total de mujeres que sobreviven a un aborto inseguro, 
5 millones requieren ser hospitalizadas para tratar complicaciones relacionadas con este(3). En cuanto al tema de la mortalidad por aborto inseguro, en términos mundiales se reporta un promedio de 47.000 mujeres al año(2).

La OMS también señala que en 2008, en Sudamérica, se realizó un total de 2.990 .000 abortos no seguros, con un resultado de 700 muertes maternas. Asimismo, se calcula que por cada mil mujeres entre 15 y 44 ańos se practican 32 abortos no seguros(4).

Cabe señalar que estos datos adolecen de dos debilidades; por un lado, los informes entregados se realizan cada ocho años, por eso las cifras no se encuentran actualizadas al año en curso; por lo mismo, sería interesante reevaluar esta información al año 2016. Por otro lado, algunos de los datos solo se pueden estimar basándose en aproximaciones, ya que donde el aborto provocado se encuentra penalizado - como ocurre en Chileesta situación únicamente puede ser valorada de manera indirecta.

Respecto de lo informado por Chile, las últimas cifras entregadas por el MINSAL indican que en 2008, de un total de 319.856 egresos hospitalarios por embarazo, parto y puerperio, los egresos por embarazo terminado en aborto representan el 10,5\% (33.428). De estos los más frecuentes fueron: otros productos anormales de la concepción (40,1\%), aborto no especificado $(33,1 \%)$, aborto espontáneo $(14,3 \%)(5)$. Estas cifras corresponden a una fracción de la realidad, ya que en ellas solo figuran aquellas mujeres que asistieron finalmente a un recinto hospitalario producto de alguna complicación, pues cifras no oficiales indican que "continúan produciéndose en Chile alrededor de 200.000 abortos ilegales al año” (6).

Junto a lo anterior, se debe tener en cuenta que unas 33 millones de usuarias de anticonceptivos quedarán embarazadas mientras usan anticoncepción(7). En consecuencia, estos embarazos tienen dos caminos; el aborto o el nacimiento de un niño no planificado. Lo anterior confirma la sentencia de que la penalización de la práctica del aborto no elimina su necesidad; por el contrario, aumenta la práctica de abortos bajo condiciones de riesgo, con el incremento respectivo de mortalidad y morbilidad que un procedimiento como este conlleva. La afirmación anterior se ve reforzada con lo declarado en 1967 por la Asamblea Mundial de la Salud, la cual catalogó el aborto inseguro como un problema de salud pública.

¿Qué opina la ciudadanía chilena acerca de esto? Para conocerlo se cuenta con el Estudio de opinión pública sobre el aborto de FLACSO 2009, el cual, dentro de nuestro país, arrojó los siguientes resultados: $86,8 \%$ de los encuestados percibe el aborto como un problema grave en el país y $63,4 \%$ como uno de salud pública, esto último en respuesta al hecho de que de cada 10 mujeres que abortan 2,8 mueren(8).

Respecto de las condiciones de realización de aborto en Chile, un $87,7 \%$ opina que no existe un trato digno hacia las mujeres que abortan(8).

Al consultar por las circunstancias en que se debiese permitir el aborto en Chile, los resultados arrojan: $66,7 \%$ por violación, $64,4 \%$ cuando se encuentra en peligro la vida de la madre, $64 \%$ por malformación del feto, $58 \%$ por incesto, $39,8 \%$ por razones de salud mental de la madre y $15 \%$ por cualquier razón que la mujer decida(8). Estos resultados muestran como el nivel de aprobación en torno a la práctica del aborto varía en función de las circunstancias, porque este será aceptado o rechazado de acuerdo al contexto en el cual se produzca.

Estas cifras no solo dejan al descubierto la problemática sanitaria de los abortos provocados en condiciones de riesgo, sino también los conflictos morales detrás de aquellos, tales como el trato indigno dispensado a las mujeres que abortan. Respecto de este último punto es conveniente aclarar que los problemas morales son parte inherente a la vida misma, y el aborto constituye uno de los tantos que la sociedad debe enfrentar. La práctica ha demostrado que es imposible resolver dichos problemas a través de la aplicación directa o casi matemática de los principios en cuestión. Porque cuando un problema moral se presenta hay una competición de valores positivos que piden su realización de manera simultánea. En el caso particular del aborto, por un lado se pide respetar y proteger el valor de la vida del que está por nacer, y, por otro, también se pide respetar el derecho a 
la autodeterminación de la mujer. Lógicamente, resulta impracticable la realización de aquellos de manera paralela y, por tanto, es imprescindible ponderar las consecuencias y circunstancias, con el fin de elegir la opción que lesione menos los valores no atendidos. Por ello, transitar desde la comprensión abstracta de la mujer a la concreta, teniendo en cuenta las circunstancias en las cuales se desarrolla el embarazo, parece ser un punto crucial para determinar en qué situaciones concretas permitir el aborto.

\section{Género y aborto}

Valdebenito y cols. indican que "la representación social de la mujer en Latinoamérica está vinculada al éthos cristiano occidental, que se caracteriza por considerar al humano - y especialmente a la mujer - como un ser de naturaleza pecadora; en consecuencia, debe sufrir constantemente el castigo y el sacrificio. Estos se expresan notoriamente a través de la represión de la sexualidad y, más aún, de la sexualidad femenina" (9). Hasta hace poco la mujer sometida al dominio del hombre asumía la sexualidad como un área remitida exclusivamente al ámbito de la reproducción y sobre cuyos resultados no siempre tenía el control deseado. La negativa de la pareja a utilizar medidas de protección, dificultad para acceder a métodos anticonceptivos de calidad, fallas en los mismos y violaciones, por mencionar algunos, son hechos que impiden un manejo efectivo de la propia fertilidad. Paralelamente, como indica Faúndez y Barzelatto, "las leyes restrictivas condenan habitualmente solo a la mujer y al proveedor, pero excepcionalmente también al hombre responsable del embarazo no deseado"(10). Más aún, hasta no hace mucho el reconocimiento de un hijo por parte del hombre era un acto de buena voluntad y no una obligación legal. No eran pocos los hombres que, frente a un embarazo, podían decidir si permanecer o huir de este, dejando a la mujer sola y a cargo de una nueva vida sin ninguna otra opción que la de asumir el nacimiento del hijo, o bien recurrir a un aborto provocado en condiciones de riesgo - el cual se da preferentemente en aquellas mujeres deprimidas socioeconómicamente-, colocando en jaque la propia vida y salud. Por ello se afirma que "es necesario eliminar las barreras regulatorias, políticas y programáticas que obstaculizan el acceso a la atención para un aborto sin riesgos y su prestación oportuna"(4), medidas que tienen como base el respeto a los derechos humanos y necesidades sanitarias.

\section{Embrión:`es poseedor de derechos?}

Pareciera que la conflictividad generada en torno al aborto se sustenta principalmente por la imposibilidad de establecer fehacientemente cuándo el nuevo ser humano en formación es persona. Frente a esta situación los discursos son múltiples y la posibilidad de llegar a una verdad generalizable y absoluta no parece posible, porque la cuestión del ser pertenece al campo de la metafísica y no de la ciencia, y en estos ámbitos solo podemos llegar a verdades prudentes y probables.

Faúndez y Barzelatto indican que las posturas van desde aquellas que "sostienen que la célula que resulta de la fertilización del óvulo ya es poseedora de plenos derechos, hasta el otro extremo de los que sostienen que la mujer tiene total autonomía para decidir sobre su cuerpo hasta el término del embarazo" (10). Ante ello, lo más prudente parece ser una postura moderada, la cual establece que "el embrión no es una persona desde el momento de la fecundación, sino que es una realidad que se va constituyendo a lo largo del tiempo hasta adquirir cualidades sistémicas nuevas, lo que exige reconocer el valor especial que esta realidad tiene" (11). Esta postura, fundamentada por Feito(11), sostiene que el embrión tiene la posibilidad de ser persona si confluyen una serie factores. Los avances en medicina embrionaria han demostrado que el programa genético no es suficiente y las interacciones extragenéticas resultan tan importantes como lo genético. Por lo tanto, si el embrión no es persona propiamente tal no se le podría asignar dignidad, ya que la dignidad es algo propio de las personas y, en consecuencia, tampoco derechos, porque aquel no tiene la característica de personalidad necesaria para portarlos y tampoco la capacidad para ejercerlos y defender intereses. Sin embargo, lo anterior no significa que se pueda rebajar al embrión a la categoría de cosa, porque tiene la posibilidad de llegar a ser persona si se dan las condiciones necesarias para ello, lo que lo dota de un especial valor: no se trata de una cosa valiosa sino de algo bueno, y por este motivo los embriones tendrán ciertos derechos, pero solo como grupo de "individuos" (11). 
Entonces, si la dignidad involucra obligaciones de respeto, y la de los bienes o entidades de valor exigen obligaciones de protección frente al daño, el embrión se situaría entre ambos, porque no es "ni cosa ni persona (...) el embrión no puede ser considerado persona, en sentido estricto, por tanto no es un fin en sí; pero tampoco es un objeto o bien material, mero medio"(11).

Por ello, cuando se presenta el clásico conflicto entre el respeto a la autodeterminación de la mujer y la obligación de protección del no nacido, surge la pregunta sobre cuál derecho y qué valor privilegiar, hasta dónde y por qué, ¿la no maleficencia de la madre o la del embrión? Chávez, citando a Gracia, plantea que en "la fase constituyente ( 6 a 8 semanas de vida) tenemos deberes imperfectos hacia un embrión que no ha alcanzado la suficiencia constitucional y, por tanto, su personeidad cabal. Los embriones en fase constituyente son sujetos de derechos, pero no los derechos de un ser humano ya constituido. Tienen un derecho genérico a la existencia, pero carecen de derechos individualmente considerados" (12). Estos deberes imperfectos se enmarcarían en el nivel 2 definido por Diego Gracia, el cual pertenece al ámbito de la gestión privada, en donde el sujeto define su proyecto de vida felicitante, la también llamada "ética de máximos" de Cortina. En tanto, en "la fase de suficiencia constitucional, en la cual tenemos deberes perfectos hacia el embrión que, habiendo alcanzado la sustantividad, es plenamente una persona humana" (12), las obligaciones se definen dentro del nivel 1, las que se desarrollan en el área de la gestión pública o la también llamada "ética de mínimos" de Adela Cortina. Los antecedentes anteriores fundamentan el sistema de plazos que presentan los distintos países donde el aborto se encuentra despenalizado. Dicho sistema establece que a menor desarrollo del embrión mayor primacía de los intereses de la madre y viceversa.

Por otra parte, la misma Constitución chilena reafirmaría esta postura moderada. Primeramente porque en su artículo 19 establece que "la Constitución asegura a todas las personas el derecho a la vida y a la integridad física y psíquica de la persona”(13). Acá se observa que la Constitución habla de personas, no de fetos, ni de embriones. $\mathrm{Y}$ aunque si bien también garantiza el derecho a la vida del no nacido, tipificando al aborto como delito, aquel se encuentra dentro del ítem del atentado contra el orden de las familias y no en el de las personas. Asimismo, la pena por el delito de aborto es menor a la pena por homicidio, lo que evidencia que el legislador considera de mucho mayor gravedad dar muerte a una persona que a un feto(14).

\section{Despenalización del aborto en Chile, un asunto de justicia}

Los antecedentes expuestos respecto del sistema de plazos para permitir el aborto, se ven reforzados y fundamentados por la dinámica cultural que guía a las sociedades actualmente. El pluralismo social propio de esta época rechaza la imposición de algún particular sistema de valores al momento de formular leyes o políticas que afectan a la sociedad en su conjunto. Esa imagen de un mundo con contenido definido y cerrado, propio de tiempos atávicos, resulta impracticable e insostenible hoy. Beauchamp y Childress apoyan lo anterior al decir que "cuando se emplean principios o reglas morales para formular o criticar políticas públicas, no se puede afirmar que el acto $x$ es moralmente correcto (o incorrecto) y deducir, por tanto, que la ley o norma y es moralmente correcta (o incorrecta) ya que obliga a/o favorece (prohíbe) el acto $x$ (...). Por ejemplo, podemos argumentar consistentemente que la esterilización o el aborto son moralmente incorrectos sin por ello suponer que la ley deba prohibirlos o negar fondos públicos a aquellas personas que de otro modo no puedan permitirse recurrir a dichos procedimientos" (15).

Al analizar el tema del aborto desde las políticas públicas chilenas se observa que estas se vuelven injustas, y a veces deshumanizadas, cuando intentan establecer leyes sobre la base de principios morales absolutos, sin reflexionar acerca de las circunstancias o el contexto de los afectados por las normas. El juicio en situación, que frente a la rigurosidad de la ley es capaz de percibir la particularidad diferenciadora en cada caso concreto, permitirá una sociedad más justa y solidaria. Esta fue la razón por la cual Italia, pese a ser un país eminentemente católico, despenalizó esta práctica para contrarrestar la gran tasa de abortos clandestinos que presentaba su nación. 
Beauchamp y Childress definen "justicia distributiva" como la "distribución igual, equitativa y apropiada en la sociedad, determinada por normas justificadas que estructuran los términos de la cooperación social. Su ámbito incluye las políticas que asignan diversos beneficios y cargas, tales como la propiedad, los recursos, los impuestos, los privilegios y las oportunidades" (15). De acuerdo con esta definición, negar el acceso al aborto seguro atenta contra este principio, ya que, por una parte, no se reconocen derechos sexuales y reproductivos de la mujer enmarcados dentro del contexto de los derechos humanos ${ }^{2}$, los cuales, por pertenecer a la categoría de derechos humanos, no requieren mérito alguno por parte del receptor, y por otra se produce una falla en la distribución de las cargas en contra de las mujeres menos favorecidas, producto de la lotería social de la vida, ya que el aborto provocado en condiciones de riesgo se da preferentemente en mujeres deprimidas socioeconómicamente. Aquello contradice el espíritu del principio de justicia, el cual, de acuerdo con Rawls, establece que la distribución de los beneficios sociales admite la presencia de desigualdades, siempre y cuando estas se orienten al aumento de las cuotas de bienestar de los menos favorecidos.

Ahora bien, la penalización del aborto no solo lesiona el principio de justicia desde la perspectiva de la equidad, sino también el principio formal de justicia, el cual declara que "los iguales deben ser tratados igualmente, y los desiguales deben ser tratados desigualmente" (15). Por lo mismo, "las decisiones, las reglas y las leyes tienden a ser injustas cuando hacen distinciones entre clases de personas que son realmente similares en aspectos relevantes"(15). Y si bien se podrá decir que efectivamente en Chile todas las mujeres embarazadas que solicitan un aborto son tratadas igualmente, negándose a todas ellas el procedimiento, también todos saben que las mujeres con ingresos más elevados podrán optar a intervenciones seguras aunque estas se encuentren al margen de la

\footnotetext{
${ }^{2}$ Que han sido legitimados internacionalmente a través de las distintas declaraciones respecto del tema: Convención Internacional sobre la eliminación de todas las formas de discriminación racial contra la mujer, 1969; Pacto Internacional de Derechos Económicos, Sociales y Culturales, 1976; Pacto Internacional de Derechos Civiles y Políticos, 1976; Convención sobre la eliminación de la discriminación contra la mujer, 1981; Convención contra la tortura y otros tratos o penas crueles, inhumanos o degradantes, 1987, etc.
}

ley, ya porque viajan a otros países para proveerse de los servicios, o bien porque los procedimientos son realizados en clínicas privadas del propio país bajo la denominación de otro acto quirúrgico. En tanto las mujeres con menores ingresos tienen una sola opción para interrumpir su embarazo: el aborto provocado en condiciones de riesgo. En consecuencia, el factor socioeconómico constituye un elemento relevante de desigualdad que obliga a tratar desigualmente. No obstante, dicho trato desigual debe ir en beneficio de aquellas personas menos aventajadas, por lo mismo no basta con la despenalización de esta práctica, sino que también es imprescindible garantizar dichas prestaciones dentro de la cartera en los servicios públicos de salud.

Y si al principio formal de justicia, que dice que los iguales deben ser tratados iguales, se aplican los principios materiales de justicia - aquellos que "especifican las características relevantes para un tratamiento igual porque identifican las propiedades sustantivas para la distribución" (15)—, como son por ejemplo las necesidades fundamentales, o sea, aquellas necesidades que, de no ser cubiertas, podrían generar un daño o perjuicio fundamental en la persona, entonces, desde este punto de vista, si negar el acceso al aborto seguro podría atentar contra la vida o la salud de la mujer, se podría considerar como una necesidad fundamental, que por ser tal requiere ser otorgada a todas las mujeres que lo necesiten.

Por otro lado, si además se atiende a las propiedades relevantes de las involucradas, o sea, "aquellas propiedades que hacen que la persona que las posee esté cualificada para optar a una distribución particular"(15), esto ocasionaría que la pobreza, la vulnerabilidad y la falta de oportunidades constituyan propiedades a las que prestar especial atención como motivo de justificación ante el requerimiento obligatorio de dicho procedimiento en los sistemas públicos de salud en Chile. Ya que son precisamente las mujeres pobres las que están más impedidas de acceder a procedimientos seguros, dada su precariedad económica.

Y si bien estos principios materiales y formales de la justicia pueden resultar muy abstractos frente a la complejidad de los temas que levanta la problemática del aborto, en general lo que se busca 
con ellos no es otra cosa que contrarrestar el elemento azaroso de la vida que conlleva a formas injustas de distribución, en otras palabras, aspirar a la regla de la oportunidad justa, la que declara que "nadie debe tener garantizados beneficios sociales sobre la base de condiciones ventajosas no merecidas (porque ninguna persona es responsable de tenerlas) y que a nadie se deberían negar beneficios sociales sobre la base de condiciones desventajosas no merecidas (porque tampoco son responsables de esas propiedades)" (15). Por lo tanto, si no está en las manos de las personas elegir, rechazar o superar dicha situación, en este caso el embarazo, sin comprometer la salud o la vida, no es justo negar la oportunidad de acceso a un aborto seguro.

En consecuencia, al penalizar el aborto se estarían negando directamente oportunidades sociales a las menos favorecidas económicamente, pues aunque el aborto se encuentre penalizado en Chile el abanico de opciones para sortear dicha dificultad es directamente proporcional al ingreso. Por consiguiente, la manera de equiparar la situación consistiría en eliminar las barreras restrictivas y establecer claramente las condiciones y requisitos para acceder a un aborto seguro en los tiempos y plazos correspondientes. Porque la regla de la oportunidad justa declara que "a todos se les dé una oportunidad justa en la vida cuando sufren desventajas que no está en su mano evitar" (15), como por ejemplo haber nacido mujer y pobre.

Además, la literatura indica que "los países con leyes que restringen severamente el acceso al aborto legal y seguro, violan los principios de no maleficencia y beneficencia, ya que estas restricciones solo aumentan el daño que sufren las mujeres y la sociedad, sin disminuir el número de los abortos"(10). Las cifras confirman esta necesidad y la sociedad en todo el mundo así lo exige. Cook y cols. indican que "los países que se suscribieron en 1995 a la declaración de Beijing invitan a considerar la revisión de las leyes que contienen medidas punitivas contra la mujer que se somete a un aborto ilegal. El análisis también solicita a los países que penalizan a las mujeres que se someten o intentan abortos a reformar sus respectivas leyes"(16). La Asamblea Mundial de la Salud afirmó en 2004: "el aborto inseguro, una causa prevenible de mortalidad y morbilidad maternas, debe abordarse como parte del Objetivo de Desarrollo del Tercer Milenio relativo a la mejora de la salud materna y de otros objetivos y metas internacionales de desarrollo" (4). Como explica Dides: "si bien la reproducción humana y sus consecuencias constituyen asuntos del ámbito privado, relacionados con la vida afectiva de las personas, se convierten en un tema político cuando su ocurrencia hace surgir la necesidad de elaborar políticas públicas"(17).

Por otra parte, si el Estado opta por prohibir a sus ciudadanos el acceso a determinados procedimientos sanitarios, tiene la obligación de entregar una alternativa válida a la cual recurrir. Si el Estado chileno dice "prohíbo el aborto", también debe garantizar a todas aquellas mujeres que no quieren o no pueden tener a su hijo el cuidado y la custodia de este. Como indica Grabiner, en "condiciones que favorezcan el desarrollo de este último en el contexto de una calidad de vida aceptable, educación, salud, hogar, un cuidado básico que todo individuo necesita para el desarrollo de sus potencialidades"(18), situación que dista mucho de la actual. Kottow señala que "las sociedades no se han abocado a paliar los problemas resultantes del nacimiento de nińos condenados a la pobreza y a la desnutrición, ni han elaborado infraestructuras que permitan a seres seriamente discapacitados recibir un nivel de cuidados, educación e inserción social que les permita un grado aceptable de bienestar" (7). Si el Estado solo se encarga de prohibir el aborto y no responsabilizarse por las consecuencias de sus dictámenes, incurre en una beneficencia a medias, que termina finalmente en una actitud maleficente.

Es necesario reconocer y garantizar los derechos sexuales y reproductivos de la mujer dentro del marco del respeto a los derechos humanos. El control y ejercicio de la maternidad involucra también la opción de interrumpir un embarazo cuando la mujer lo estime conveniente. Briozzo indica que "el respeto a la autonomía de la mujer en su decisión, sea cual fuere, implica una concepción de democracia y equidad y es un elemento imprescindible para la construcción de una sociedad más libre. El respeto de los derechos no obliga a nadie hacer algo que no quiera. Por el contrario, la falta de respeto obliga a cometer delitos y con ello exponerse a riesgos y daños"(19). 
Con todos estos argumentos, en ningún momento se está promoviendo ni estimulando la realización de abortos; por el contrario, el panorama ideal sería aquel en que ninguna mujer tuviese que experimentar aquello. Los participantes de la conferencia del Cairo están de acuerdo en que "no se debería promover el aborto como un método regular de planificación familiar, la prevención de embarazos no deseados debiera tener siempre una alta prioridad"(16). Es necesaria una integración de las distintas esferas de la vida reproductiva, desde educación sexual, planificación familiar y acceso al aborto seguro, todas ellas condiciones básicas de un sistema de salud equitativo y justo. Por esto, lo único que persigue la despenalización de esta conducta es reconocer la libertad de la mujer en el manejo de su vida reproductiva, cuyo control no puede estar condicionado por el nivel socioeconómico, ni por el sistema moral de terceras personas. Pero para llegar a esto ha de ser necesario, como dice Chávez, el diálogo abierto, el cual "es el único modo de enfrentar la pregunta en el marco del principio absoluto del respeto hacia todas las personas en torno a decisiones que les afecten. Aunque no pueda obtenerse un marco de consenso respecto a lo que sea bueno, al menos podría acordarse lo que sea justo para todos"(12).

\section{Conclusiones}

Al hablar de aborto es común pensar en los movimientos pro-life y pro-choice. Sin embargo, estas visiones resultan extremas y no definen a la población en general; más aún, muchas veces dificultan el debate al no aceptar ninguna solución intermedia.

La opinión pública chilena exige una revisión de las leyes en torno a la figura del aborto, en pro de una despenalización de esta práctica, con el fin de paliar los problemas social y sanitario que los abortos ilegales e inseguros generan, y con ello tratar de disminuir la discriminación que experimentan las mujeres pobres y vulnerables, pues son ellas las que sufren mayoritariamente el peso de la ley.

En pleno siglo XXI no es posible imponer visiones particulares de mundo al resto de la sociedad, y es necesario, por medio del discurso de todos los implicados, llegar a un consenso. La perspectiva de género ha de ser incorporada — ya que el embarazo y el aborto se producen en el cuerpo de la mujer - y también la voz ciudadana, porque las políticas públicas afectan a toda la comunidad; por consiguiente, solo una auténtica deliberación logrará una verdadera democratización del tema.

El Estado necesita generar políticas públicas que establezcan los límites en los cuales estas prácticas estén permitidas, pues es parte de su deber asegurar el acceso a la salud y libertad de sus ciudadanos para la consecución de sus proyectos de vida y felicidad. El derecho al aborto seguro, concebido como un derecho de las mujeres en el ámbito de la salud sexual y reproductiva, es algo que Chile pide y el mundo apoya como un constituyente más de la justicia social. 


\section{Referencias}

1. Organización Panamericana de la Salud. Derogación del Aborto Terapéutico en Nicaragua: Impacto en Salud. Nicaragua: OPS.

2. World Health Organization. Unsafe abortion. Global and regional estimates of the incidence of unsafe abortion and associated mortality in 2008. Geneva: WHO; 2011.

3. Haddad LB, Nour NM. Unsafe Abortion: Unnecessary Maternal Mortality. Reviews in Obstetrics \& Gynecology 2009; 2(2): 122-126.

4. World Health Organization. Unsafe abortion incidence and mortality. Global and regional levels in 2008 and trends during 1990-2008. WHO; 2012.

5. Ministerio de Salud. Orientaciones técnicas para la atención integral de mujeres que presentan un aborto y otras pérdidas reproductivas. Santiago de Chile: MINSAL; 2011.

6. Kottow M. Introducción a la bioética. $2^{\circ}$ ed. Santiago de Chile: Mediterráneo; 2005.

7. Organización Mundial de la Salud. Aborto sin riesgos: guía técnica de politicas para sistemas de salud. Ginebra: OMS; 2012.

8. Dides C, Benavente MC, Sáez I. Principales Resultados Estudio de Opinión Pública sobre Aborto: Brasil, Chile, México, Nicaragua. Serie Documentos Electrónicos Programa inclusión Social y Género 2010; 1.

9. Valdebenito C, Lama A, Lolas F. Relación mujer y biotecnología: Aproximación al impacto de la bioética. Acta Bioethica 2006; 12(2): 145-150.

10. Faúndez A, Barzelatto J. Ética y aborto. En: Escríbar A, Pérez M, Villarroel R, editores. Bioética. Fundamentos y Dimensión Práctica. Santiago de Chile: Mediterráneo; 2004: 287-303.

11. Feito L. estatuto del embrión humano: cuestiones filosóficas y consideraciones morales. En: Beca JP, Astete C, editores. Bioética Clínica. Santiago de Chile: Mediterráneo; 2012: 281-297.

12. Chávez P. Discusión en torno al estatuto ontológico y ético del embrión. En: Escríbar A, Pérez M, Villarroel R, editores. Bioética. Fundamentos y Dimensión Práctica. Santiago de Chile: Mediterráneo; 2004: 226-256.

13. Ministerio Secretaria General de la Presidencia. Constitución Politica de la República de Chile. Santiago de Chile: 1980.

14. Vivanco A. Aspectos jurídicos del llamado "aborto terapéutico" en Chile. ARS Médica 2001; 4(6): 154-179.

15. Beauchamp T, Childress J. Principios de Ética Biomédica. $4^{\circ}$ ed. Barcelona: Masson; 2002.

16. Cook R, Dickens B, Bliss L. International Developments in Abortion Law from 1988 to 1998. Health Law and Ethics 1999; 89(4): 579-586.

17. Dides C. Aportes al debate sobre el aborto en Chile: derechos, género y Bioética. Acta Bioethica 2006; 12(2): $219-229$.

18. Grabiner G. A Question of Choice. Reproductive Health 2011; 8(20): 7.

19. Briozzo L. Aborto provocado: un problema humano. Perspectivas para su análisis-Estrategias para su reducción. Rev Med Uruguay 2003; 19: 188-200.

Recibido: 21 de julio de 2014

Aceptado: 11 de agosto de 2014 